\title{
Commensal gut microbes play an important role in shaping host physiology
}

\begin{abstract}
The bacteria residing in the gut are physiologically important to the health and wellness of the human beings. It is imperative to investigate the interaction pathway between the gut microbes as several studies have shown that when the delicate microbial balance is impacted, it often leads to dysbiosis. The microbial metabolites secreted by the microbes are chemical messengers interacting with the host component in the lumen. The onset of several autoimmune disorders of the gastro-intestinal tract has been attributed to the abundance of some of these metabolites present as above normal concentrations under physiological homeostasis.
\end{abstract}

Keywords: microbiome, homeostasis, dysbiosis, autoimmune disorders, gastrointestinal tract
Volume I Issue 2 - 2015

Somdutta Saha
Computational Biology, R\&D, GlaxoSmithKline, USA

Correspondence: Somdutta Saha, Computational Biology, R\&D, GlaxoSmithKline, USA, Email Somdutta.x.saha@gsk.com

Received: July 21, 2015 | Published: November 18, 2015

\section{Introduction}

Commensal microbes colonizing the human gastro-intestinal (GI) tract are responsible for shaping the host's physiology in many ways. The microbial "organ" helps in proper development of the intestine, processing of nutrients, protection from exogenous pathogens, and maturation of the immune system ${ }^{1}$ and in return the host provides a nutrient-rich environment to the microbial population. However, when the delicate balance in this ecosystem is disturbed, physiological homeostasis is impacted upon, often resulting in dysbiosis. Dysbiosis or the state of 'disturbed homeostatsis' has now been clinically related to the onset of several autoimmune diseases like inflammatory bowel disease, Crohn's disease, multiple sclerosis, asthma, type 1 diabetes, cancers $^{2-4}$ where the composition of the gut microbiota has been seen to be altered from various metagenomic studies. The onset of psoriatic arthritis, which is a chronic inflammation of the joint, is often associated with the chronic inflammatory skin condition- psoriasis, is still increasing. These studies suggest that drastic shifts in microbial ecosystems or dysbiosis impact disease pathology through specific host-microbial interaction pathways.

\section{Microbial metabolites have important immune-mo- dulatory properties}

Very few microbes are known whose immune-modulatory properties have been explored- prominent amongst these areFaecalibacterium prausnitzii and Prevotella copri. ${ }^{5,6}$ A microbial byproduct- equol, ${ }^{7}$ have demonstrated anti-inflammatory and anticancer function for G-protein coupled receptor 109A (Gpr109A) in colon. Gpr109A is the receptor for butyrate and niacin, a pharmacological agonist of Gpr109A. Butyrate, a bacterial product from the fermentation of dietary fiber in colon, has been implicated as the key mediator of anti-inflammatory and anti-tumorigenic effects. Other short chain fatty acids (SCFAs) such as acetate, propionate, hexaoate are also known to possess immuno- regulatory activities. Apart from SCFAs, the microbiota secretes metabolites including organic acids, bacteriocins, peptides, and others, which influence aspects of cell differentiation, programmed cell death, cell proliferation, angiogenesis and cancer development. For example, bacterial lipopolysaccharides
(LPS) induce the expression of immune cytokines that, in turn, affect host immune response. ${ }^{8}$ On the other hand, bacterial lactic acid reduces pro-inflammatory responses in intestinal epithelial cells. ${ }^{9}$

Antimicrobial peptides, including defensins, are essential effectors in host defense and in the maintenance of immune homeostasis. Clinical studies have linked the defective expression of both $\alpha$ and $\beta$-defensin to the reduced killing of certain microorganisms by the intestinal mucosa of patients suffering from ileal and colonic Crohn's disease (CD), respectively. Defensins may promote the formation of micelles by electrostatic forces to negatively-charged components of the microbial membrane, including LPS from Gram negative bacteria and lipoteichoic acids from Gram positive bacteria. Conversely, the Gram positive bacteria Staphylococcus epidermidis and Staphylococcus aureus are thought to repulse the killing activity of defensins by expressing a membrane-bound molecule with a high density of negative charge ${ }^{8}$ and by modifying its lipid membrane through the lipid-modifying Multiple Peptide Resistance Factor (Mprf), ${ }^{9}$ respectively. Similarly, both the two-component system PhoP/ PhoQ and lipopolysaccharide are involved in resistance to defensins in the facultative intracellular bacterium Salmonella typhimurium, ${ }^{10}$ providing a mechanism whereby certain pathogens may circumvent innate immune mechanisms. Additional investigations are now eagerly awaited to determine whether luminal secretion of certain defensins may influence immune homeostasis in any part of the gastrointestinal tract.

Many endogenous metabolites interact with host receptors. For example, bacterial LPS is continuously released in the gut when the Gram-negative bacteria die. LPS is then physiologically translocated into intestinal capillaries through a toll-like receptor (TLR) 4-dependent mechanism. ${ }^{10}$ The LPS reaches the target tissues from the intestine by a mechanism facilitated by lipoproteins, notably chylomicrons freshly synthesized from epithelial intestinal cells in response to a high-fat diet. ${ }^{11}$ Additionally, it also triggers the secretion of proinflammatory cytokines when it binds to the complex of mCD14 and the TLR4 at the surface of innate immune cells. ${ }^{12,13}$ Thus, it was demonstrated that LPS is an important initial factor for the incidence of high-fat diet-induced metabolic diseases. Lamichhane et al., ${ }^{14}$ 
have reported a high-throughput approach for identifying essential metabolites of an organism using genetic and biochemical approaches and then implement computational approaches to identify metabolite mimics.

Apart from the primary metabolites produced by commensal bacterial there are secondary metabolites or metabolite- derivatives that shape host physiology or play an important beneficial role. Neoechinulin A, an antioxidant compound, suppresses lipid oxidation, useful for protecting against neuronal cell death in neurodegenerative diseases. ${ }^{15}$ Another microbial metabolite isolated from the fungal strain Penicillium sp., Epolactaene, arrests the cell cycle at the G0/G1 phase and induces the outgrowth of neurites in human neuroblastoma SHSY5Y cells. ${ }^{16}$ 20-O- $\beta$-(D-glucopyranosyl)-20(S)-protopanaxadiol or compound $\mathrm{K}$ extracted from Gingseng has chemo-preventive effects in colonic tumorigenesis. ${ }^{17}$ Also phenolic derivatives or benzoic esters secreted by Clostridium difficile, F. prausnitzii, Bifidobacterium, Subdoligranulum, Lactobacillus etc. are elevated in various diseases and some of them can be used as biomarker for certain diseases like hypertension and obesity, colorectal cancer and autism. ${ }^{18,19}$ Thus the microbial population and its secreted metabolites are also very significant from a disease prognosis and incidence point of view.

\section{Discussion}

The human body is the habitat of trillions of microbes and the symbiotic relationship that exists between them is the secret to healthy living. The knowledge of the importance of this relationship has led to the opening of new avenues in scientific discoveries, exploring the co-evolution of the host-microbiome for many pathophysiological events. ${ }^{20-22}$ Studies have shown that modulating the composition of the microbes to restore the healthy state from its diseased state can be an effective way to find newer treatment regimes. The microbial genome associated with the human body is an integral component of the comprehensive view on pharmaco-genomics. A mechanistic understanding of how the gut microbiota directly and indirectly affects drug metabolism is beginning to emerge, ${ }^{23}$ and is rapidly expanding. Different types of probiotic bacteria are present in the gut and convey varying health benefits to the intestine. Probiotics must be ingested regularly for any health-promoting activity to persist. It is possible to manipulate (at least temporarily) the composition of the intestinal microflora through dietary supplementation with probiotics. An ideal probiotic should possess most of the following characteristics: cell adherence capability, multiply, survive, and produce hydrogen peroxide acids, bacteriocins against pathogenic growth, be safe, non-invasive, non-carcinogenic, non-pathogenic in nature and coaggregate to form a normal balanced flora. Presently, there is lack of knowledge on how the gut microbiome profile affects the response to an ingested drug. The microbiome may affect gene expression by host tissues that, in turn, may affect drug pharmacodynamics. Thus, a thorough investigation of the microbial population in the host microenvironment is essential for bridging the unmet need in treating autoimmune disorders of the GI tract.

\section{Acknowledgements}

None.

\section{Conflict of interest}

The author declares no conflict of interest.

\section{References}

1. Hooper LV, Gordon JI. Commensal host-bacterial relationships in the gut. Science. 2001;292(5519):1115-1118.

2. de Goffau MC, Luopajarvi K, Knip M, et al. Fecal microbiota composition differs between children with beta-cell autoimmunity and those without. Diabetes. 2013;62(4):1238-1244.

3. Morgan XC, Tickle TL, Sokol H, et al. Dysfunction of the intestinal microbiome in inflammatory bowel disease and treatment. Genome Biol. 2012;13(9):R79.

4. Xuan C, Shamonki JM, Chung A, et al. Microbial dysbiosis is associated with human breast cancer. PLoS One. 2014;9(1):e83744.

5. Scher JU, Sczesnak A, Longman RS, et al. Expansion of intestinal Prevotella copri correlates with enhanced susceptibility to arthritis. Elife. 2013;2:e01202.

6. Sokol H, Pigneur B, Watterlot L, et al. Faecalibacterium prausnitzii is an anti-inflammatory commensal bacterium identified by gut microbiota analysis of Crohn disease patients. Proc Natl Acad Sci U S A. 2008;105(43):16731-16736.

7. Singh N, Gurav A, Sivaprakasam S, et al. Activation of Gpr109a, receptor for niacin and the commensal metabolite butyrate, suppresses colonic inflammation and carcinogenesis. Immunity. 2014;40(1):128139.

8. Audy J, Mathieu O, Belvis J, et al. Transcriptomic response of immune signalling pathways in intestinal epithelial cells exposed to lipopolysaccharides, Gram-negative bacteria or potentially probiotic microbes. Benef Microbes. 2012;3(4):273-286.

9. Carey CM, Kostrzynska M. Lactic acid bacteria and bifidobacteria attenuate the proinflammatory response in intestinal epithelial cells induced by Salmonella enterica serovar Typhimurium. Can J Microbiol. 2013;59(1):9-17.

10. Neal MD, Leaphart C, Levy R, et al. Enterocyte TLR4 mediates phagocytosis and translocation of bacteria across the intestinal barrier. $J$ Immunol. 2006;176(5):3070-3079.

11. Vreugdenhil AC, Rousseau CH, Hartung T, et al. Lipopolysaccharide (LPS)-binding protein mediates LPS detoxification by chylomicrons. $J$ Immunol. 2003;170(3):1399-1405.

12. Sweet MJ, Hume DA. Endotoxin signal transduction in macrophages. $J$ Leukoc Biol. 1996;60(1):8-26.

13. Wright SD, Ramos RA, Tobias PS, et al. CD14, a receptor for complexes of lipopolysaccharide (LPS) and LPS binding protein. Science. 1990;249(4975):1431-1433.

14. Lamichhane G, Freundlich JS, Ekins S, et al. Essential metabolites of Mycobacterium tuberculosis and their mimics. MBio. 2011;2(1):e00301e00310.

15. Maruyama K, Ohuchi T, Yoshida K, et al. Protective properties of neoechinulin A against SIN-1-induced neuronal cell death. J Biochem. 2004;136(1):81-87.

16. Nagumo Y, Kakeya H, Yamaguchi J, et al. Structure-activity relationships of epolactaene derivatives:structural requirements for inhibition of Hsp60 chaperone activity. Bioorg Med Chem Lett. 2004;14(17):4425-4429.

17. Dougherty U, Mustafi R, Wang Y, et al. American ginseng suppresses Western diet-promoted tumorigenesis in model of inflammationassociated colon cancer:role of EGFR. BMC Complement Altern Med. 2011;11:111. 
18. Lord RS, Bralley JA. Clinical applications of urinary organic acids. Part 2. Dysbiosis markers. Altern Med Rev. 2008;13(4):292-306.

19. Zheng X, Xie G, Zhao A, et al. The footprints of gut microbialmammalian co-metabolism. J Proteome Res. 2011;10(12):5512-5522.

20. Holmes E, Li JV, Marchesi JR, et al. Gut microbiota composition and activity in relation to host metabolic phenotype and disease risk. Cell Metab. 2012;16(5):559-564.
21. Dave M, Higgins PD, Middha S, et al. The human gut microbiome: current knowledge, challenges, and future directions. Transl Res. 2012;160(4):246-257.

22. de WT, Dore J, Lepage P. Does our food (environment) change our gut microbiome ('in-vironment'):a potential role for inflammatory bowel disease? Dig Dis. 2012;30(Suppl 3):33-39.

23. Haiser HJ, Turnbaugh PJ. Is it time for a metagenomic basis of therapeutics? Science. 2012;336(6086):1253-1255. 